\title{
EFEITO ANALGÉSICO DO BUTORFANOL NA DOR SOMÁTICA EM GATOS ANESTESIADOS COM PROPOFOL
}

\author{
ANALGESIC EFFECT OF BUTORPHANOL ON SOMATIC PAIN IN CATS \\ ANESTHETIZED WITH PROPOFOL
}

\author{
Isabela Ciniello Araujo ${ }^{1}$ Luiz Gonzaga Pompermayer ${ }^{2}$ Fernanda Antunes ${ }^{3}$ \\ Almir Pereira de Souza ${ }^{3}$ Marco Aurélio Ferreira Lopes ${ }^{4}$
}

\section{RESUMO}

O propofol é um agente anestésico intravenoso usado para indução e manutenção da anestesia, mas produz analge sia limitada, havendo a necessidade do uso concomitante de analgésicos. Avaliou-se o efeito analgésico do butorfanol na dor somática em gatos anestesiados com doses fracionadas de propofol. Foram utilizados 16 animais, distribuídos aleatoriamente em dois grupos. Os animais do grupo controle foram pré-tratados com 0,2mg/kg de acepromazina por via IM e, após 15 minutos, receberam $6 \mathrm{mg} / \mathrm{kg}$ de propofol por via IV. Os animais do grupo tratamento foram pré-medicados com uma combinação de acepromazina $(0,2 \mathrm{mg} / \mathrm{kg})$ e butorfanol $(0,8 \mathrm{mg} / \mathrm{kg})$, administrados na mesma seringa por via IM, e, após 15 minutos, receberam $6 \mathrm{mg} / \mathrm{kg}$ de propofol por via IV. Em ambos os grupos, a manutenção da anestesia foi feita com administrações de propofol, na dose de $3 \mathrm{mg} / \mathrm{kg}$, por via IV, sempre que necessário, durante 60 minutos. A necessidade de readministração de propofol foi verificada pela resposta apresentada ao pinçamento cutâneo, através de uma pinça de Kocher. Avaliaram-se também as freqüências cardíaca e respiratória, pressão arterial média, saturação de oxiemoglobina e temperatura retal. A administração de butorfanol causou apenas redução nas freqüências cardíaca e respiratória e na saturação de oxiemoglobina, em comparação com o grupo controle, sem exercer influência significativa sobre o período hábil, a dose total administrada e o período de recuperação do propofol. Concluiu-se que a adição de butorfanol não produziu analgesia somática em gatos anestesiados com doses fracionadas de propofol.

Palavras-chave: gato, felino, anestesia, propofol, butorfanol.

\section{SUMMARY}

Propofol is an intravenous anesthetic agent used for induction and maintenance of anesthesia but produces limited analgesia, and concomitant use of analgesics is necessary. The analgesic effect of butorphanol in somatic pain in cats anesthetized with intermittent doses of propofol was evaluated. Sixteen animals were randomly assigned to 2 groups. Control group animals were premedicated with IM acepromazine $(0,2 \mathrm{mg} / \mathrm{kg})$ and after 15 minutes IV propofol $(6 \mathrm{mg} / \mathrm{kg})$ was administered. Treatment group animals were premedicated with IM acepromazine $(0,2 \mathrm{mg} / \mathrm{kg})$ and butorphanol $(0,8 \mathrm{mg} / \mathrm{kg})$, mixed in the same syringe and after 15 minutes IV propofol $(6 \mathrm{mg} / \mathrm{kg})$ was administered. In both groups anesthesia was maintained with repeated injections of propofol $(3 \mathrm{mg} / \mathrm{kg})$ as needed, during 60 minutes. The need to complement propofol doses was determined by reactions to a skin pinch with a Kocher hemostatic forceps. Heart rate, respiratory rate, mean blood pressure, rectal body temperature and oxyhemoglobin saturation were also recorded. Administration of butorphanol caused minimal changes in cardiopulmonary variables compared to control group and did not affect duration of anesthesia and total dose of propofol or recovery period. We concluded that addition of butorphanol did not produce somatic analgesia during anesthesia maintained with repeated injections of propofol in cats.

Key words: cat, feline, anesthesia, propofol, butorphanol.

\section{INTRODUÇÃO}

O propofol é um alquilfenol com propriedades sedativas/hipnóticas semelhantes às dos tiobarbitúricos. Não apresenta, porém, efeito cumulativo após administrações repetidas, podendo ser utilizado através de administrações intermitentes ou infusão contínua, sem efeito significativo no tempo

\footnotetext{
${ }^{1}$ Aluna de Especialização, Departamento de Veterinária, Universidade Federal de Viçosa (UFV), Viçosa, MG.

${ }^{2}$ Professor, Departamento de Veterinária, UFV, 36571-000, Viçosa, MG. E-mail: lgonzaga@ mail.ufv.br. Autor para correspondência.

${ }^{3}$ Alunos de Pós-graduação - Departamento de Veterinária, UFV, Viçosa, MG.

${ }^{4}$ Professor, Departamento de Veterinária, UFV, Viçosa, MG.
} 
ou na qualidade da recuperação. Além disso, a aplicação perivascular de propofol não produz reação inflamatória local severa (GLEN, 1980; WATKINS et al., 1987; MAMA, 1998). Como a analgesia produzida por esse fármaco é limitada, é necessário o uso concomitante de analgésicos (MAMA, 1998).

O butorfanol é um analgésico opióide kappa agonista-Mu antagonista sintético, sendo sete vezes mais potente que a morfina, mas com menor tendência a causar depressão respiratória. Tem sido usado em cães e gatos na indução de anestesia e em combinação com agentes anestésicos para potencializar a analgesia (HOSGOOD, 1990).

Os analgésicos opióides podem produzir efeitos indesejáveis em gatos, como excitação motora, alucinações e convulsões. Durante anos, o termo "morfinomania" foi utilizado para descrever esses efeitos dos opióides nos felinos (MUIR \& SWANSON, 1994). Entretanto, estudos feitos com o uso de morfina demonstraram que a estimulação é conseqüência da superdosagem e que doses mais baixas do que as recomendadas para cães podem ser usadas com segurança nesta espécie (DAVIS \& DONNELLY, 1968; HEAVNER, 1970; WATTS $\boldsymbol{e}$ t al., 1973).

Poucos estudos farmacológicos têm sido feitos em felinos e, em geral, os conhecimentos obtidos com a pesquisa em cães são extrapolados para os gatos. No entanto, deve-se considerar que os felinos apresentam muitas particularidades na biotransformação ou nos efeitos de diversas classes de drogas, entre elas os fenóis e os opióides (EVANS, 1988).

Este experimento teve como objetivos avaliar o efeito analgésico do butorfanol na dor somática de gatos pré-tratados com acepromazina e anestesiados com doses fracionadas de propofol, bem como verificar os efeitos dessa associação de fármacos sobre as variáveis clínicas e fisiológicas dessa espécie.

\section{MATERIAL E MÉTODOS}

Foram utilizados 16 gatos sem raça definida, de ambos os sexos, clinicamente normais. Os felinos foram mantidos em baias, recebendo ração comercial balanceada ${ }^{\mathrm{a}}$ para a espécie e água à vontade. Os gatos foram aleatoriamente distribuídos em dois grupos de 8 animais (grupos controle e tratamento) e deixaram de receber ração e água 12 horas antes do início do experimento.

Nos animais do grupo controle, foi feita a aplicação de $0,2 \mathrm{mg} / \mathrm{kg}$ de acepromazina ${ }^{\mathrm{b}}$ por via intramuscular (IM), seguida da aplicação de propofol $^{\mathrm{c}}, 15$ minutos depois, na dose de $6 \mathrm{mg} / \mathrm{kg}$, por via intravenosa (IV), durante 60 segundos. Os animais do grupo tratamento receberam $0,2 \mathrm{mg} / \mathrm{kg}$ de acepromazina e $0,8 \mathrm{mg} / \mathrm{kg}$ de butorfanol ${ }^{\mathrm{d}}$, na mesma seringa, por via IM, seguida da aplicação de propofol, 15 minutos depois, na dose de $6 \mathrm{mg} / \mathrm{kg}$, por via IV, durante 60 segundos. Em ambos os grupos, a anestesia foi mantida durante 60 minutos com propofol, na dose de $3,0 \mathrm{mg} / \mathrm{kg}$, por via IV, administrado sempre que necessário, através de um dispositivo intravenoso $23 \mathrm{G}$, introduzido na veia cefálica. Foi utilizada uma bomba de infusão ${ }^{e}$ para administração de solução fisiológica, na velocidade de $10 \mathrm{~m} \ell / \mathrm{kg} / \mathrm{h}$, para manter um volume circulatório adequado durante a anestesia.

As variáveis avaliadas foram: 1- temperatura retal, medida com termômetro digital f; 2freqüência respiratória, obtida pela contagem dos movimentos da parede torácica durante um minuto; 3- frequiência cardíaca, com o uso de um oxímetro de pulso $^{\mathrm{g}}$, com o sensor colocado sobre a pele previamente depilada na porção proximal da cauda; 4pressão arterial média (PAM), medida indiretamente, no membro pélvico direito, imediatamente acima da articulação fêmuro-tíbio-patelar, com um monitor de pressão não invasivo ${ }^{\text {h }}$; 5- saturação da oxiemoglobina $\left(\mathrm{SpO}_{2}\right)$ obtida com um oxímetro de pulso; 6- analgesia, avaliada através do pinçamento de uma prega cutânea em quatro locais pré-determinados (base da cauda, face lateral do abdome, face lateral do tórax e face lateral do pescoço) a cada 3 minutos, utilizando-se uma pinça hemostática de Kocher e aplicando-se pressão até alcançar o primeiro dente da cremalheira. A resposta do animal ao estímulo (movimento em qualquer parte do corpo, aumento da frequiência cardíaca ou respiratória) ou a ausência de resposta indicou a necessidade ou não de complementação da dose de propofol; 7- miorrelaxamento, avaliado de acordo com o grau de relaxamento da musculatura abdominal e dos membros e classificado em ausente, moderado ou intenso; 8período hábil do propofol, definido como o tempo compreendido entre o fim da administração da primeira dose do propofol e a administração da segunda dose (primeira dose complementar); 9- período de recuperação, definido como o tempo compreendido entre o término do tempo experimental e o momento que o animal adquiriu posição quadrupedal; 10- dose total administrada, obtida pela soma de todas as doses administradas durante o tempo experimental .

As variáveis foram avaliadas em 4 momentos: 15 minutos após a administração da medicação pré-anestésica (MPA), imediatamente após a administração de propofol, 30 e 60 minutos após a administração de propofol.

Foi feita análise de perfil (MORRISON, 1967; CURI, 1980) para as variáveis mensuradas em 
todos os momentos. Os dados referentes a período hábil, período de recuperação, número de repetições de propofol e dose total administrada foram avaliados pela análise de variância para experimento inteiramente aleatório, seguidos pelo teste " $\mathrm{t}$ " de Student. Em todos os casos, valores de $\mathrm{p}<0,05$ foram considerados estatisticamente significativos.

\section{RESULTADOS E DISCUSSÃO}

Quinze minutos após a administração da MPA, os animais de ambos os grupos apresentaramse tranqüilizados, permitindo o manuseio com facilidade. Nenhum gato tratado com opióide apresentou qualquer sinal de excitação, como os descritos por MCKELVEY \& HOLLINGSHEAD (1994a) e MUIR \& SWANSON (1994). Sabe-se que as fenotiazinas bloqueiam os receptores dopaminérgicos (BOOTH, 1992; BREARLEY, 1994), os quais estão envolvidos no aparecimento de excitação no gato (PENNEY \& WHITE, 1978; BOOTH, 1992; MACY, 1994; WILCKE, 1994). Assim, o uso da acepromazina na MPA pode ter contribuído para a prevenção dos efeitos excitatórios do opióide.

O período hábil médio foi de 6 minutos no grupo controle (GC) e de 8 minutos no grupo tratamento (GT), e a dose total média de propofol para os animais do GC foi $26 \mathrm{mg} / \mathrm{kg}$ e para o GT foi $24 \mathrm{mg} / \mathrm{kg}$ (Tabela 1). Não houve diferença estatisticamente significativa entre os grupos para essas variáveis, o que indica que o butorfanol não produziu analgesia somática nos gatos anestesiados com propofol. SAWYER \& RECH (1987) observaram que o butorfanol promove analgesia somática com a dose de $0,8 \mathrm{mg} / \mathrm{kg}$ por via IV, idêntica à dose usada no presente experimento, por via IM. Esperava-se uma menor dose total e um maior período hábil nos animais tratados com butorfanol, pois, segundo SHORT \& BUFALARI (1999), a medicação pré-

Tabela 1 - Valores médios e desvios-padrões (entre parênteses) do período hábil da primeira dose (minutos), período de recuperação (minutos) e dose total de propofol ( $\mathrm{mg} / \mathrm{kg}$ ) em gatos anestesiados com propofol, pré-tratados com acepromazina (grupo controle) ou com acepromazina e butorfanol (grupo tratamento).

Grupo controle Grupo tratamento

\begin{tabular}{lcc}
\hline & $6(1,07) \mathrm{a}$ & $8(3,53) \mathrm{a}$ \\
Período hábil da primeira dose & $105(21,33) \mathrm{b}$ & $137(47,95) \mathrm{b}$ \\
Período de recuperação & $26(5,5) \mathrm{c}$ & $24(3,4) \mathrm{c}$ \\
Dose total de propofol & & \\
\end{tabular}

Para cada variável, médias seguidas pela mesma letra na linha não diferem significativamente pelo teste t de Student $(\mathrm{p}<0,05)$. anestésica com opióides reduz a dose de propofol necessária para a indução e manutenção anestésicas em pequenos animais.

O período médio de recuperação para os animais do grupo controle foi de 105 minutos, enquanto para os animais tratados com butorfanol foi de 137 minutos (Tabela 1), não havendo diferença estatisticamente significativa entre os grupos. Esses resultados contrariam as afirmações de SHORT \& BUFALARI (1999), de que o uso de tranqüilizantes ou analgésicos na medicação pré-anestésica prolonga o período de recuperação do propofol em pequenos animais. Os tempos médios de recuperação foram bastante elevados em ambos os grupos, diferindo dos valores de 37 e 27 minutos observados por outros autores (BREARLEY et al., 1988; MORGAN \& LEGGE, 1989). Embora a literatura relate ausência de efeito cumulativo do propofol (GLEN, 1980; WATKINS et al., 1987; MAMA, 1998) e MORGAN \& LEGGE (1989) não tenham observado relação entre o número de doses administradas e o tempo de recuperação em cães e gatos, os resultados encontrados no presente experimento sugerem efeito cumulativo da droga. Tal efeito também foi sugerido no estudo de ANDRESS et al. (1995), que atribuíram o aumento, nos períodos de recuperação, à deficiência dos gatos em metabolizar compostos fenólicos e à inibição das enzimas microssomais hepáticas pelo propofol. Durante o período de recuperação, dois gatos do GC e três do GT apresentaram espirros, e a maioria dos animais de ambos os grupos esfregou o focinho com a pata. Segundo BREARLEY et al. (1988), MORGAN \& LEGGE (1989) e ANDRESS et al. (1995), essas manifestações são normalmente observadas no período de recuperação quando o propofol é utilizado em gatos.

A temperatura retal dos animais comportou-se de modo semelhante nos dois grupos. Observou-se uma diminuição gradativa ao longo do tempo experimental e não houve diferença significativa entre os grupos em nenhum momento (Tabela 2). Segundo MUIR \& HUBBELL (1992) e MCKELVEY \& HOLLINGSHEAD (1994b), vários fatores contribuem para a redução da temperatura corporal durante a anestesia geral, tais como: depressão do sistema termorregulador central, menor atividade metabólica, vasodilatação periférica e a incapacidade de produzir calor através da atividade muscular esquelética. A administração da acepromazina também pode ter contribuído para esse resultado, pois os agentes fenotiazínicos inibem a reatividade do termostato hipotalâmico, permitindo uma redução na temperatura corporal (GUYTON \& HALL, 1996). 
Tabela 2 - Valores médios e desvios-padrões (entre parênteses) da temperatura retal (TR), frequiência cardíaca (FC), freqüência respiratória (FR), pressão arterial média (PAM) e saturação de oxiemoglobina (SpO2) de gatos anestesiados com propofol, pré-tratados com acepromazina (grupo controle) ou com acepromazina e butorfanol (grupo tratamento).

\begin{tabular}{|c|c|c|c|c|c|c|c|c|}
\hline & \multicolumn{2}{|c|}{15 min após a MPA } & \multicolumn{2}{|c|}{$\begin{array}{c}\text { Logo após a } \\
1^{-} \text {dose de propofol }\end{array}$} & \multicolumn{2}{|c|}{$\begin{array}{c}30 \text { min após a } \\
1^{-} \text {dose de propofol }\end{array}$} & \multicolumn{2}{|c|}{$\begin{array}{c}60 \text { min após } \\
1^{\underline{a}} \text { dose de propofol }\end{array}$} \\
\hline & Controle & Tratamento & Controle & Tratamento & Controle & Tratamento & Controle & Tratamento \\
\hline $\mathrm{TR}^{\circ} \mathrm{C}$ & $\begin{array}{l}38,2 \mathrm{a} \\
(0,3)\end{array}$ & $\begin{array}{l}38,6 \mathrm{a} \\
(0,6)\end{array}$ & $\begin{array}{l}37,8 \mathrm{~b} \\
(0,4)\end{array}$ & $\begin{array}{l}38,1 \mathrm{~b} \\
(0,6)\end{array}$ & $\begin{array}{l}36,3 \text { c } \\
(0,4)\end{array}$ & $\begin{array}{l}36,5 \mathrm{c} \\
(1,0)\end{array}$ & $\begin{array}{l}35,3 \mathrm{~d} \\
(0,5)\end{array}$ & $\begin{array}{l}35,4 \quad \mathrm{~d} \\
(1,2)\end{array}$ \\
\hline $\mathrm{FC}$ & 230 a & 204 a & 192 & 169 & 152 & 129 & $140 \mathrm{~b}$ & $120 \mathrm{~b}$ \\
\hline $\mathrm{Bat} / \mathrm{min}$ & $(23,0)$ & $(40,7)$ & $(7,8)$ & $(26,9)$ & $(5,1)$ & $(29,5)$ & $(10,3)$ & $(28,5)$ \\
\hline FR & $41 \mathrm{a}^{*}$ & $40 \quad \mathrm{a}$ & $32 \mathrm{~b}^{*}$ & $25 \mathrm{~b}$ & $21 \#$ & $17 \&$ & $19 \#$ & $14 \&$ \\
\hline Mov/min & $(9,4)$ & $(8,8)$ & $(4,9)$ & $(9,1)$ & $(2,1)$ & $(4,7)$ & $(2,1)$ & $(3,4)$ \\
\hline $\begin{array}{l}\text { PAMJ } \\
\text { MmHg }\end{array}$ & $\begin{array}{c}82 \quad \mathrm{a} \\
(12,4)\end{array}$ & $\begin{array}{c}90 \quad \mathrm{a} \\
(114,5)\end{array}$ & $\begin{array}{l}60 b^{*} \\
(17,8)\end{array}$ & $\begin{array}{c}67 \quad b \# \\
(19,2)\end{array}$ & ${ }_{(18,5)}^{56} \mathrm{c} \#$ & $\begin{array}{l}51 \\
(10,4)\end{array}$ & $\underset{(13,6)}{55} \mathrm{~d}^{*}$ & $\begin{array}{l}59 \\
(9,9)\end{array}$ \\
\hline $\begin{array}{l}\mathrm{SpO}_{2} \\
\%\end{array}$ & $\begin{array}{l}97 \quad \mathrm{a} * \\
(2,4)\end{array}$ & $\begin{array}{l}94 \text { a \# } \\
(4,0)\end{array}$ & $93 b^{*}$ & 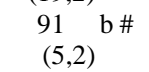 & ${ }_{(6,5)}^{92} \mathrm{c}^{*}$ & ${ }_{(6,9)}^{88} \mathrm{c} \#$ & $\begin{array}{l}94^{*} \\
(4,5)\end{array}$ & $\begin{array}{l}88 \# \\
(4,7)\end{array}$ \\
\hline
\end{tabular}

Para cada variável, médias seguidas pela mesma letra ou símbolo na linha não diferem significativamente pela análise de perfil (p<0,05). As letras representam a igualdade entre os grupos em cada momento, e os símbolos a igualdade entre os momentos dentro de cada grupo.

A freqüência cardíaca comportou-se de forma semelhante nos dois grupos, apresentando perfis similares, mas não iguais. Os valores do GC foram significativamente maiores do que os do GT imediatamente após a administração de propofol e 30 minutos depois (Tabela 2). A comparação dos momentos dentro de cada grupo revelou diminuição significativa e gradativa em ambos. A bradicardia observada nos dois grupos era esperada, pois, segundo MAGELLA \& CHEIBUB (1990), WATERMAN \& LUCKE (1994) e MAMA (1998), o propofol produz depressão cardiovascular semelhante ou mais intensa que a observada com o tiopental. A redução da freqüência cardíaca mais pronunciada nos animais tratados com butorfanol está de acordo com o descrito por TRIM (1983), que observou diminuição na freqüência cardíaca em cães após a administração desse opióide. Segundo HALL \& CLARKE (1991), os opióides causam bradicardia, provavelmente por estímulo vagal.

A pressão arterial média comportou-se de forma igual nos dois grupos, havendo uma redução significativa imediatamente após a administração do propofol e manutenção dos valores durante todo o tempo experimental. (Tabela 2). Esses resultados estão de acordo com as informações de GLEN (1980), NOCITE $\boldsymbol{e t}$ al. (1990) e DUKE (1995). Ao contrário da maioria dos agentes indutores, que provocam uma taquicardia compensatória, como os barbitúricos, a diminuição da pressão causada pela aplicação de propofol não afeta de maneira reflexa a freqüência cardíaca (GLEN, 1980; MARSICO et al., 1991).
Segundo SEBEL \& LOWDON (1989), a hipotensão provocada pelo propofol é mais pronunciada que a produzida pela maioria dos agentes anestésicos, inclusive o tiopental, sendo devida a vários mecanismos, tais como: vasodilatação periférica, diminuição do tono simpático, ações cronotrópica e inotrópica negativas e depressão do reflexo barorreceptor (YANG et $\boldsymbol{a l}$., 1997). WEAVER \& RAPTOPULOS (1990) estudaram a indução de anestesia com propofol em cães e gatos e observaram queda na pressão arterial no momento da indução, porém com retorno aos níveis normais com o passar do tempo. No presente experimento, a manutenção da pressão arterial em níveis baixos por todo o tempo experimental, provavelmente deve-se a doses repetidas do propofol visando ao prolongamento do período anestésico. Segundo MAGELLA \& CHEIBUB (1990), os opióides podem potencializar a hipotensão produzida pelo propofol. Entretanto, de acordo com AMEER \& SALTER (1979), ao contrário da morfina, o butorfanol causa apenas uma pequena redução na pressão arterial. Deve-se considerar, também, que tanto os opióides (MAGELLA \& CHEIBUB, 1990) como as fenotiazinas (HALL \& CLARKE, 1991) são drogas que reduzem a pressão arterial devido a seus efeitos sobre o tono vascular periférico (CORNICK \& HARTSFIELD, 1992).

A freqüência respiratória comportou-se de maneira igual nos dois grupos. Observou-se que os valores do GC foram significativamente maiores que os do GT 30 minutos e 60 minutos após a aplicação do propofol. A comparação entre momentos, dentro de cada grupo, revelou que no GC a freqüência res- 
piratória foi significativamente menor 30 minutos após a primeira dose de propofol e no GT imediatamente e 30 minutos após a administração de propofol (Tabela 2). A saturação de oxiemoglobina comportou-se de modo semelhante nos dois grupos, apresentando perfis similares, mas não iguais. A saturação de oxiemoglobina do GC foi significativamente maior 60 minutos após a aplicação de propofol e não se observou diferença significativa entre os momentos dentro de cada grupo (Tabela 2). O propofol pode causar depressão respiratória por atuar diretamente sobre a atividade inspiratória central e a resposta ventilatória à $\mathrm{PaCO} 2$ (SMITH, et $\boldsymbol{a l} .$, 1993). Os analgésicos opióides podem produzir depressão respiratória severa (MCKELVEY \& HOLLINGSHEAD, 1994a), mas o butorfanol, por suas propriedades kappa agonista e $\mathrm{Mu}$ antagonista, produz depressão limitada (ROSOW, 1986; HOSGOOD, 1990). Neste experimento, observou-se depressão respiratória em ambos os grupos, sendo que o pré-tratamento com butorfanol promoveu uma maior redução, tanto da freqüência respiratória como da saturação da oxiemoglobina. Esses resultados estão de acordo com as observações de MAGELLA \& CHEIBUB (1990), de que o uso concomitante de analgésicos opióides pode potencializar a depressão respiratória durante o período de manutenção da anestesia com propofol.

Nenhum animal apresentou apnéia durante a indução com propofol, o que contradiz os achados de outros estudos, de que a apnéia é uma observação comum (WATKINS et al., 1987; MORGAN \& LEGGE, 1989; NOCITE et al., 1990; SMITH et al., 1993) e que ocorre em todos os pacientes que recebem um opióide na MPA (MAGELLA \& CHEIBUB, 1990). Para evitar o aparecimento de apnéia durante a fase de indução, adotou-se, neste experimento, um tempo relativamente longo (1 minuto) para a administração do propofol. Segundo alguns autores (GLEN, 1980; MARSICO et al, 1991; MAMA, 1998), a administração lenta de propofol evita a manifestação de apnéia.

Imediatamente após a indução com propofol, e em todos os momentos subseqüentes, o miorrelaxamento foi considerado intenso para todos os animais, de ambos os grupos. Segundo GLEN (1980) e DUKE (1995), o relaxamento muscular é uma característica notável do propofol, embora MARSICO et al. (1991) relatem um miorrelaxamento mínimo no cão.

Concluiu-se, com base nos resultados apresentados, que o butorfanol utilizado no GT, além de não produzir analgesia somática nos gatos anestesiados por propofol, causou desoxigenação arterial (SpO2 < 90\%), após 30 minutos de aneste- sia, e que ambos os protocolos utilizados causaram hipotensão arterial e período de recuperação prolongado.

\section{FONTES DE AQUISIÇÃO}

a - Catshow - Purina Nutrimentos Ltda - Brasil

b - Acepran 1\% - Univet S. A. - Brasil

- Diprivan - Zeneca Farmacêutica do Brasil Ltda - Brasil

d - Torbugesic - Fort Dodge Laboratories, Inc. - USA

e - Bomba de Infusão FARS 600 - Lifemed Pesquisas Médicas Ind. E Com. Ltda - Brasil

f - Termômetro Digital B-D - Brasil

g - Nellcor ${ }^{\circledR}$ N-100 Pulse Oximeter - USA

h - Biomonitor 4 - Bio Engenharia de Sistemas e Equip. SA Brasil

\section{REFERÊNCIAS BIBLIOGRÁFICAS}

AMEER, B., SALTER, F.J. Drug therapy reviews: Evaluation of butorphanol tartrate. Am J Hosp Pharm, v.36, p.1683-1691, 1979.

ANDRESS, J.L., DAY, T.K., DAY, D. The effects of consecutive day propofol anesthesia on feline red blood cells. Vet Surg, v.24, n.3, p.277-282, 1995.

BOOTH, N.H. Neuroleptoanalgésicos, analgésicos narcóticos e antagonistas analgésicos. In: BOOTH, N. H., MCDONALD, L. E. Farmacologia e terapêutica em veterinária. 6. ed Rio de Janeiro: Guanabara Koogan, 1992. Cap.15, p.231261.

BREARLEY, J.C., KELlAGHER, R., HALL, L.W. Propofol anaesthesia in cats. J Small Anim Pract, v.29, p.315-322, 1988.

BREARLEY, J.C. Sedation, premedication and analgesia. In HALL, L.W., TAYLOR, P.M. Anaesthesia of the cat. London : Baillière Tindall, 1994. Chapter 7, p.111-128.

CORNICK, J.L., HARTSFIELD, S.M. Cardiopulmonary and behavioral effects of combinations of acepromazine / butorphanol and acepromazine / oxymorphone in dogs. J Am Vet Med Assoc, v.200, n.12, p.1952-1956, 1992.

CURI, P.R. Análise de medidas repetidas em experimentos biológicos. Rev Bras Estatística, v.41, p.137-150, 1980.

DAVIS, L.E., DONNELLY, E.J. Analgesic drugs in the cat. J Am Vet Med Assoc, v.153, n.9, p.1161-1167, 1968.

DUKE, T. A new intravenous anesthetic agent: Propofol. Can Vet J, v.36, p.181-183, 1995.

EVANS, R.J. Terapêutica. In: CHANDLER, E.A., HILBERY, A.D.R., GASKELL, C.J. Medicina e terapêutica de felinos. 2 ed. São Paulo : Manole, 1988. Cap.27, p.419-436.

GLEN, J.B. Animal studies of the anaesthetic activity of IC 35868. Brit J Anaeth, v.52, p.731-742, 1980.

GUYTON, A.C., HALL, J.E. Textbook of medicine physiology. 9.ed. Philadelphia : Saunders, 1996. Chapter 75: Body temperature, temperature regulation, and fever: p.911-922.

HALL, L.W., CLARKE, K.W. Veterinary anaesthesia. 9.ed. London : Baillière Tindall, 1991. Chapter 4: Principles of sedation, analgesia and premedication: p.51-79. 
HEAVNER, J.E. Morphine for postsurgical use in cats. J Am Vet Med Assoc, v.156, n.8, p.1018-1019, 1970.

HOSGOOD, G. Pharmacologic features of butorphanol in dogs and cats. J Am Vet Med Assoc, v.196, n.1, p.135-136, 1990.

MACY, D.W. Pharmacologic considerations in the cat. Feline Pract, v.22, n.2, p.14-18, 1994

MAGELLA, H.A., CHEIBUB, Z.B. Propofol: revisão bibliográfica. Rev Bras Anestesiol, v.40, n.4, p.289-294, 1990.

MAMA, K. New drugs in feline anesthesia. Comp Contin Educ Pract Veter, v.20, n.2, p.125-139, 1998.

MARSICO, F., TENDILLO, F.J., GÓMEZ DE SEGURA, I.A., $\boldsymbol{e}$ al. Un nuevo anestésico intravenoso: propofol. Evaluación clínica y experimental en el perro. Clin Vet Peq Anim, v.11, n.4, p.231-239, 1991

MCKELVEY, D., HOLlingSHEAD, K.W. Small animal anesthesia canine and feline practice. St. Louis : Mosby, 1994a. Chapter 1: the preanesthetic period: p.1-54

MCKELVEY, D., HOLLINGSHEAD, K.W. Small animal anesthesia canine and feline practice. St. Louis : Mosby, 1994b. Chapter 2: general anesthesia: p.55-118.

MORGAN, D.W.T., LEGGE, K. Clinical evaluation of propofol as an intravenous anaesthetic agent in cats and dogs. Vet Rec, v.124, p.31-33, 1989

MORRISON, D.F. Multivariate statistical methods. New York: McGrows Hill, 1967. 388p.

MUIR, W.W., HUBBELL, J.A.E.. Manual de anestesia veterinária. Zaragoza : Acribia, 1992. Cap.16: vigilancia del paciente durante la anestesia: p.189-197.

MUIR, W.W., SWANSON, C.R. Principles and techniques of anesthesia and chemical restraint. In: SHERDING, R.G. The cat diseases and clinical management. 2.ed. Philadelphia: Saunders, 1994. Chapter 5, p.91-132.

NOCITE, J.R., SERZEDO, P.S.M.M., ZUCULOTTO, E.B., et al Características clínicas da indução anestésica e da intubação traqueal com propofol. Rev Bras Anestesiol, v.40, n.6, p.385-390, 1990

PENNEY, B.E., WHITE, R.J. Narcotic analgesics in the domestic cat. Vet Clin North Am: Small Anim Pract, v.8, n.2, p.317-324, 1978
ROSOW, C.E. Butorphanol in perspective. Acute Care, v.12 (suppl 1), p.2-7, 1986

SAWYER, D.C., RECH, R.H. Analgesia and behavioral effects of butorphanol, nalbuphine, and pentazocine in the cat. J Am Anim Hosp Assoc, v.23, p.438-446, 1987.

SEBEL, P.S., LOWDON, J.D. Propofol: A new intravenous anesthetic. Anesthesiology, v.71, p.260-277, 1989.

SHORT, C.E., BUFALARI, A. Propofol anesthesia. Vet Clin North Am: Small Anim Pract, v.29, n.3, p.747-778, 1999.

SMITH, J.A., GAYNOR, J.S., BEDNARSKI, R.M., $\boldsymbol{e t}$ al. Adverses effects of administration of propofol with various preanesthetic regimens in dogs. J Am Vet Med Assoc, v.202 n.7, p.1111-1115, 1993.

TRIM, C.M. Cardiopulmonary effects of butorphanol tartrate in dogs. Am J Vet Res, v.44, n.2, p.329-331, 1983.

WATERMAN, A.E., LIVINGSTON, A., AMIN, A. Analgesic activity and respiratory effects of butorphanol in sheep. Res Vet Sci, v.51, p.19-23, 1991.

WATERMAN, A.E., LUKE, J.N. Anestesia intravenosa. In HILBERY, A.D.R. Manual de anestesia de los pequeños animales. 2.ed. Zaragoza : Acribia, 1994. Cap.6, p.45-56.

WATKINS, S.B., HALL, L.W., CLARKE, K.W. Propofol as an intravenous anaesthetic agent in dogs. Vet Rec, v.120, p.326329, 1987.

WATTS, S.J., SLOCOMBE, R.F., HARBISON, W.D., $\boldsymbol{e} \boldsymbol{t} \boldsymbol{a l}$. Assessment of analgesia and other effects of morphine and thiambutene in the mouse and cat. Aust Vet J, v.49, p.525529, 1973.

WEAVER, B.M.Q., RAPTOPOULOS, D. Induction of anaesthesia in dogs and cats with propofol. Vet Rec, v.126, p.617-620, 1990.

WILCKE, J.R. Principles of drug therapy. In: SHERDING, R.G. The cat diseases and clinical management. 2. ed. Philadelphia : Saunders, 1994. Chapter 3, p.25-37.

YANG, C-Y, LUK, H-N, CHEN, S-Y, et al. Propofol inhibits medullary pressor mechanisms in cats. Can J Anaesth, v.44, n.7, p.775-781, 1997.

Ciência Rural, v. 31, n. 1, 2001. 